\title{
Review on Improve Performance Localization in Wireless Sensor Networks Based Received-Signal- Strength Technique
}

\author{
Virendra Jain ${ }^{1}$, Prof.Amit Thakur ${ }^{2}$ \\ M. Tech. Scholar, Department of CSE, SVCST, RGPV, Bhopal, India ${ }^{1}$ \\ Asst. Professor, Department of CSE, SVCST, RGPV, Bhopal, India ${ }^{2}$
}

\begin{abstract}
Secure localization of unknown nodes during a Wireless Sensor Network (WSN) is a vital analysis subject. Wireless Sensor Networks (WSNs) are networks of distributed autonomous devices which will sense or monitor Physical or environmental conditions hand and glove. WSNs face several challenges, primarily caused by communication failures, with advancements in hardware style, communication protocols, resource potency, and different aspects. Recently, there has been a lot of target mobile device networks, and that we have even seen the event of small-profile sensing devices that are ready to management their own movement. though it's been shown that quality alleviates many problems with reference to device network coverage and property, several challenges stay. Among these, the necessity for position estimation is probably the foremost vital. a survey on localization strategies for mobile wireless device networks. they supply taxonomies for mobile wireless sensors and localization, as well as common architectures, measuring techniques, and localization algorithms. They conclude with an outline of real-world mobile device applications that need position estimation. Describe a 3 stage optimization technique is planned to reduce the calculable error and finding the situation of a node during a network.
\end{abstract}

Keywords: Wireless Sensor Network, security, localization, mobile sensors, anchor nodes, Localization error, received signal strength.

\section{INTRODUCTION}

Wireless Sensor Network (WSN) includes a sizable amount of detector nodes that are deployed in some areas. they will be utilized in several applications, i.e. battlefields, hearth vulnerable, security areas, observation surroundings conditions, following functions, event detection et al.. Among most of those applications, location data of detector nodes is crucial. Thus, it's necessary to get the placement data, that is termed as localization. Location identification or localization in Wireless device Network refers to estimating the position or abstraction co-ordinates of Wireless device Nodes. Localization may well be done either manually or by victimization GPS. normally the localization method equip every node with a world positioning system \&\#40;GPS\&\#41; that isn't effective thanks to size, value and power consumption constraints. The limitation of GPS is that it can't be enforced in dense forests, mountains that block the road of sight from satellites. Therefore, several localization algorithms introduced to resolve the localization drawback. Localization rule in WSN use special nodes referred to as landmarks or anchors to estimate the placement of alternative nodes employing a priori data of the special node co-ordinates. Somespecial nodes referred to as anchors being awake to their position and alternative nodes referred to as unknown nodes measures the gap from special nodes to estimate their positions. [1]

Localization is one in every of the foremost vital topics in Wireless detector Networks (WSNs) since several basic techniques in WSNs, e.g., location-based authentication need the positions of unknown nodes. Also, the positions of unknown nodes play a crucial role in several WSNs applications, like observation applications embody environmental observation, health observation, and following applications embody pursuit objects, animals, humans, and vehicles [2]. Specifically for a few applications, e.g., military applications like field of battle police work or environmental applications like fire detection, incorrect positions might cause severe consequences, e.g., wrong military selections on the field of battle and false alarms to folks thus, the problems of secure localization should be self-addressed in WSN[3]. detector network deployments are usually determined by the applying. Nodes may be placed during a grid, randomly, encompassing AN object of interest, or in incalculable alternative arrangements. In several things, AN best preparation is unknown till the 


\section{International Journal of Advanced Research in Computer and Communication Engineering}

Vol. 8, Issue 2, February 2019

detector nodes begin assembling and process information. For deployments in remote or wide areas, rearranging node positions is usually impossible. The design of economical protocols for detector networks has been a really active analysis space in recent years. A elementary drawback in designing device networks is localization - determinative the locations of the sensors. This info is helpful in several contexts - it should be used for cluster, routing and for mapping the sector being perceived. In straightforward terms, localization is mechanism for locating spatial relationship between objects. decisive the physical location of sensors when they need been deployed is thought because the drawback of localization. Sensors responsive to their position also can improve routing potency by selective flooding or selective forwarding knowledge only within the direction of the destination. detector nodes might not have AN indi vidual identifiers or addresses. the placement of the detector could also be a part of different address of the sensors. numerous algorithms that use the placement as a part of the address are propose[4]

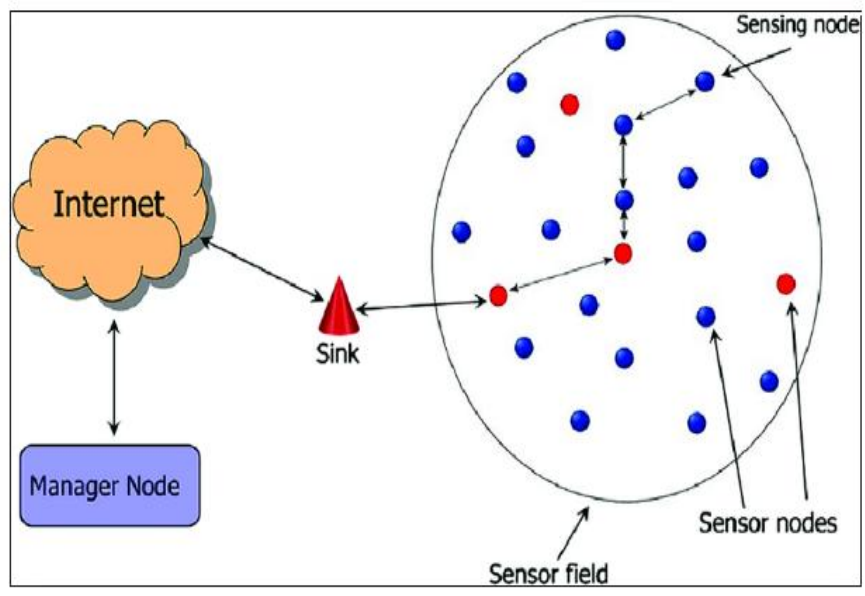

Fig1 WSN communication design

Localization is a very important analysis drawback in several systems like measuring instrument, sonar, the world positioning system, wireless networks, psychological feature radio networks transmission systems, and wireless device networks (WSNs). supply localization employing a network of sensors or wireless nodes has sweeping applications, e.g., wireless location services; search, rescue, and navigation in harsh environments; autonomous vehicles; net of Things; and watching and police work for security and defense. As a result, this analysis topic has received continuous interest and has been investigated widely [5]

\section{LITERATURE SURVEY}

In Niu et al. [6] this paper, an overview of recent developments in received-signal-strength (RSS)-based localization in wireless sensor networks is presented. Several important practical issues and their solutions are discussed. To save communication bandwidth and sensor energy, a maximum-likelihood estimator based on quantized data is presented along with its corresponding CrameÂr $\square$ Rao lower bound (CRLB) and optimal quantizer design schemes. For further system resource savings, an iterative sensor selection approach is presented to activate only the most informative sensors, by maximizing the mutual information or minimizing the posterior CRLB at each iteration. For a resource constrained WSN with imperfect wireless channels, channel-aware target localization is described, where the channel model is incorporated into the localization scheme itself, thereby improving performance without increasing communication overhead. Another practical issue involving the presence of malicious sensors called Byzantines is discussed and mitigation schemes are provided. A recent coding-theorybased approach which is both computationally inexpensive and robust to such malicious attacks is also discussed.

Harikrishnan.G et al.[7] Sensor localization has become an essential requirement for realistic applications over Wireless Sensor Networks (WSNs ). As such localization scheme designed for mobile sensor networks is necessary. Here the objective is to design a localization scheme to improve the localization accuracy by u ing localization scheme Improve $\mathrm{d}$ Monte -Carlo Localization (IMCL). In this method, all se ns or nodes are mobile, normal node collects the locations of its one -hop and two-hop anchor nodes via message exchange, and constructs a ne w possible location se $\mathrm{t}$ in e ach time $\mathrm{s}$ lot 


\section{International Journal of Advanced Research in Computer and Communication Engineering}

Vol. 8, Issue 2, February 2019

where the normal node may locate. The normal nodes without location information can estimate their own locations by gathering the positions of location -aware nodes (anchor nodes ) and the one - hop normal nodes whose locations are estimate $\mathrm{d}$ from $\mathrm{t}$ he anchor nodes. In e ach time $\mathrm{s}$ lot $\mathrm{e}$ ach normal node executes these three phases once and gets its estimate d location. Our simulation result shows that the localization error using this scheme is lower than $\mathrm{t}$ he previous schemes such as MCL, MSL and bounded box schemes under various mobility models and moving speeds.

Balasubramanian $\mathbf{S}$ et al.[8] Localization is an essential and important research issue in wireless sensor networks (WSN).Most localization schemes focus on static sensor networks.However,Mobile sensors required in some applications to acquire all the relevant dat. As such, a localization scheme defined for mobile sensor networks is necessary to track the moving nodes In this paper, we propose a localization scheme, the normal nodes without location information can estimate their own location information can estimate their own locations by gathering the positions of location aware nodes (anchor nodes) and the one-hop normal nodes whose locations are estimated from the anchor nodes. In addition, we propose a scheme that predicts the moving direction of sensor nodes to increase localization accuracy. Simulation results show that the localization error in our proposed scheme is lower than the previous schemes in various mobility models and moving speeds.

Du et al. [9] In wireless sensor networks (WSNs), sensors' locations play a critical role in many applications. Having a GPS receiver on every sensor node is costly. In the past, a number of location discovery (localization) schemes have been proposed. Most of these schemes share a common feature: they use some special nodes, called beacon nodes, which are assumed to know their own locations (e.g., through GPS receivers or manual configuration). Other sensors discover their locations based on the reference information provided by these beacon nodes. Most of the beacon-based localization schemes assume a benign environment, where all beacon nodes are supposed to provide correct reference information. However, when the sensor networks are deployed in a hostile environment, where beacon nodes can be compromised, such an assumption does not hold anymore. In this paper, we propose a general scheme to detect localization anomalies that are caused by adversaries. Our scheme is independent from the localization schemes. We formulate the problem as an anomaly intrusion detection problem, and we propose a number of ways to detect localization anomalies. We have conducted simulations to evaluate the performance of our scheme, including the false positive rates, the detection rates, and the resilience to node compromises.

In Sheu J.P et al.[10] the literature, there exist many solutions to locating moving objects based on different localization issues for wireless sensor networks (WSN). Sensor networks are expected to revolutionize information gathering, processing and dissemination in many diverse environments. Existing localization algorithms can be categorized as either range-based or range-free schemes. Range - based schemes are not suitable for WSN because of their irregularity of radio propagation and their cost of additional devices. In contrast, range-free schemes do not need to use received signal strength to estimate distances. They only need simple and cheap hardware and are more suitable for WSN. However, existing rangefree schemes are too costly and not accurate enough or are not scalable. To improve previous works, a fully distributed range-free localization scheme for WSN [12] is presented, based on the assumption that only a few sensor nodes, called anchors, know their locations, and the remaining (normal) nodes need to estimate their own locations by gathering nearby neighboring information. The improved grid-scan algorithm is used here to find the estimated locations of the normal nodes and a vector-based refinement scheme to improve the accuracy of the estimated locations.

Md. Tareq Adnan et al.[11] Localization is defined as the problem of determining the geographical locations of nodes in a wireless ad hoc or sensor network. Networks typically have some nodes (called seeds) that know their locations (using GPS or other means). Other (non-seed) nodes compute their locations by exchanging messages with nodes within their radio range. Several algorithms have been proposed for localization in different scenarios. Algorithms have been designed for networks in which each node has ranging capabilities (i.e., can estimate distances to its neighbors). Other algorithms have been proposed for networks in which no node has such capabilities. Some algorithms only work when nodes are static. Some other algorithms are designed specifically for networks in which all nodes are mobile. In this paper we propose a range-based localization algorithm RMCB for wireless sensor networks. Our algorithm uses a small fraction of seeds. It also makes use of the received signal strength measurements that are available from the sensor hardware. Our algorithm works well when nodes are static or mobile. We demonstrate using empirical data from sensor hardware (Texas Instruments EZ 430-RF2500) and simulations that RMCB outperforms a very good range-free algorithm WMCL in terms of localization error and has a similar computational complexity to WMCL. 


\title{
International Journal of Advanced Research in Computer and Communication Engineering
}

\author{
Vol. 8, Issue 2, February 2019
}

Xinwei Wanga et al. [12] A multilateration algorithm is presented in this work in order to realize a precise localization of the sensor nodes in wireless sensor networks in logistic applications. RSSI (Received Signal Strength Indicator) is used for distance measurements between sensor nodes. The developed algorithm is simulated and afterwards implemented in a real sensor network for food transportation logistic. The test results show that the multilateration algorithm with RSSI reaches the requirements of logistic applications.

B. Sriniva al. [13], was proposed that in WSN the localization was an essential issue because many applications require sensor nodes to know their locations with a high degree of precision. An optimal path planning method for the mobile anchors based on localization. The proposed path planning method determined the location of the individual sensor nodes with the help of mobile anchor nodes. It ensured that the trajectory of the mobile anchor nodes minimized the localization error and guaranteed that all of the sensor nodes could determine their locations. Then, the PSO algorithm determined the trajectory of the mobile anchor nodes. The path planning strategy method performed in both smaller localization error and a high percentage of localized sensor nodes by PSO.

S.H. Hong et al.[14] .This paper describes a localization algorithm necessary for building small-sized network's position reporting system using wireless sensor network. In existing sensor networks, much of the localization algorithm is devoted to making position estimations by learning a minimum of 3 position values of anchor node that's aware of absolute position value under the environment where sensor nodes are fixed. The proposed algorithm has an indefinite traveling direction and has shown in performance analysis that it is possible to estimate positions even with a small number of anchors. Also, by using the implemented board, although practicality has been proven with implementation of realistic distance measurement through distance measurement using RSSI(Received Signal Strength Indication) and traveling distance measurement using acceleration sensor.

R. Shubair et al.[15]. the distance-based localization techniques are surveyed for WSNs. It is impossible to present a complete review of every published algorithm. Therefore, ten representative distance-based localization algorithms that have diverse characteristics and methods are chosen and presented in detail. The authors outline a tiered classification mechanism in which the localization techniques are classified as distributed, distributed-centralized, or centralized. Generally, centralized localization algorithms produce better location estimates than distributed and distributed-centralized algorithms. However, much more energy is consumed in the centralized algorithms due to high communication overheads for packet transmission to the base station. Distributed-centralized localization algorithms are always used in cluster-based WSNs, which can produce more accurate location estimates than distributed algorithms without significantly increasing energy consumption or sacrificing scalability. Localization of sensor nodes is an important aspect in Wireless Sensor Networks (WSNs). This paper presents an overview of the major localization techniques for WSNs. These techniques are classified into centralized and distributed depending on where the computational effort is carried out. The paper concentrates on the factors that need to be considered when selecting a localization technique. The advantages and limitation of various techniques are also discussed. Finally, future research directions and challenges are highlighted.

\section{PROBLEM FORMULATION}

Localization is one among the most drawback in WSN. to resolve this downside several localization techniques are used. In WSN localization technique may be explained in 2 section that are go section \& Position section. In go section, node live their distances from the anchors victimisation several algorithms as Received signal strength, AOA (angle of arrival), TOA (time of arrival) etc. In estimation section the node estimate their position mistreatment the go data. this is often done either by mistreatment geometric approach which provides the precise resolution or by victimisation the improvement approach that reduced the error in locating the coordinates of the device node (unknown node). several researchers had approached the localization drawback from totally different perspective. Here we tend to target the localization technique supported distance measurements and reduced the localization error between the calculable location location of the device node.

\section{EXPECT OUTCOME}

In the field of research wireless sensor networks and determines several challenges. Find accuracy and minimize error in wireless sensor networks communication and optimum answer. 


\title{
International Journal of Advanced Research in Computer and Communication Engineering
}

\author{
Vol. 8, Issue 2, February 2019
}

\section{CONCLUSION}

the projected secure localization schemes. it will improve the positioning performance. and therefore the distributed algorithmic program is extremely easy and it can also work. although once the network is disconnected that's no international coordination is required among nodes and this sort of algorithmic program provides satisfactory accuracy.

There are many directions for future adds WSN localization. Reducing localization latency is one among the foremost necessary benchmarks for WSNs. Currently; a exchange exists between the speedy execution of associate algorithmic program and its accuracy. Extra work is required that targeted on reducing run-time and error, whereas maintaining positioning accuracy. Additionally, the bulk of localization algorithms to this point are centralized. For mobile detector localization, this is this can be often a poor style alternative, because of the extra error and energy prices incurred. the event of additional distributed localization techniques would be a welcome addition to WSN localization. there's a lot of interest in localization in urban and indoor areas wherever obstacles like vehicles, walls, people, and piece of furniture cause multipath propagation and loss of line of sight. Most current ways use some variation of RSS identification, in conjunction with improvement techniques. However, new ways square measure needed as they expand mobile sensing to areas wherever training information cannot safely be obtained, like urban war zones or burning buildings. Lastly, mobile motivated sensors are currently being developed with motesized kind factors. Like embedded device nodes, these devices even have resource constraints, that limit their ability to navigate a sensing region within the same means a automaton with a full array of sensors and powerful process capability would possibly. we are able to expect to check several advances in mobile device navigation within the close to future.

\section{REFERENCES}

[1] Z. Chaczko, R. Klempous, J. Nikodem and M. Nikodem, "Methods of sensors localization in wireless networks", IEEE, 2007.

[2] S. U. Sastry, N. and D. Wagner, "Secure verification of location claims," in Proceedings of the 2nd ACM workshop on Wireless security, September 2003.

[3] Y. Zeng, J. Cao, J. Hong, and L. Xie, "Secure localization and location verification in wireless sensor networks," in IEEE 6th International Conference on Mobile Adhoc and Sensor Systems, October 2009, pp. 864-869.

[4] H. Wymeersch, J. Lien, and M. Z. Win, "Cooperative localization in wireless networks," Proc. IEEE, vol. 97, no. 2, pp. 427-450, Feb. 2009.

[5] A. H. Sayed, A. Tarighat, and N. Khajehnouri, "Network-based wireless location: Challenges faced in developing techniques for accurate wireless location information," IEEE Signal Process, Mag.,vol. 22, no. 4, pp. 24-40, Jul. 2005.

[6] Niu, Ruixin, Aditya Vempaty, and Pramod K. Varshney. "Received-signal-strength-based localization in wireless sensor networks." Proceedings of the IEEE 106.7 (2018): 1166-1182.

[7]Harikrishnan.G, "Minimizing Localization Error in Wireless Sensor Networks", International Journal of Advanced Information Science and Technology (IJAIST) ISSN: 2319:2682 Vol.2, No.10, October 2013.

[8] Balasubramanian, S., and R. Kavitha. "Minimizing the localization error in wireless sensor networks." Procedia engineering 38 (2012): $3097-3104$.

[9] Du, Wenliang, Lei Fang, and P. Ningi. "LAD: Localization anomaly detection for wireless sensor networks." 19th IEEE International Parallel and Distributed Processing Symposium. IEEE, 2005.

[10] Sheu J.P, Chen P.C, and Hs u C.S, "A Dis tributed Localization Sche me for Wireles s Sensor Networks with Improved Grid-Scan and Vector-Bas ed Refine ment," IEEE Trans . Mobile Co mputing, 2008, Vol. 7, No. 9, pp. 1110 - 1123.

[11] Md. Tareq Adnan, Suprakash Datta, Stuart MacLean, "Efficient and Accurate Range-based Sensor Network Localization”, Procedia Computer Science, $405-413,10,2012$.

[12] Xinwei Wanga, Ole Bischoffa, Rainer Laura, Steffen Paula, "Localization in Wireless Ad-hoc Sensor Networks using Multilateration with RSSI for Logistic Applications", Elsevier ,Available online 4 September , 461-464,2009.

[13] B. Sriniva and P. sangeetha , "Mobile Anchor-Based localization Using PSO and Path Planning Algorithm In Wireless Sensor Networks", IJIRAS, Vol. 2, pp 5-8, 2015.

[14] Sung-Hwa Hong, Byoung-Kug Kim, and Doo-Seop Eom,"Localization Algorithm in Wireless Sensor Networks with Network Mobility” Manuscript received September 20, , IEEE, 2009.

[15]A. Kulaib, R. Shubair, M. Al-Qutayri, and J. W. Ng, "An overview of localization techniques for wireless sensor networks," in Innovations in Information Technology (IIT), International Conference on, pp. 167-172, IEEE, 2011. 